The COVIRL-001 Trial: A multicentre, prospective, randomised trial comparing standard of care (SOC) alone, SOC plus hydroxychloroquine monotherapy or SOC plus a combination of hydroxychloroquine and azithromycin in the treatment of noncritical, SARS-CoV-2 PCR-positive population not requiring immediate resuscitation or ventilation but who have evidence of clinical decline: A structured summary of a study protocol for a randomised controlled trial

Eoin Feeney ${ }^{1,2}$, Deborah Wallace ${ }^{2 *}$, Aoife Cotter ${ }^{2,3}$, Willard Tinago ${ }^{2}$, Cormac McCarthy ${ }^{1,2}$, David Keane ${ }^{1,2}$, Rabia Hussain ${ }^{2}$, Elena Alvarez Barco ${ }^{2}$, Peter Doran ${ }^{2,4}$ and Patrick Mallon ${ }^{1,2}$

\begin{abstract}
Objectives

Hydroxychloroquine has attracted considerable interest as a potential therapy for COVID-19 infection due to its immunomodulatory effects and reported efficiency in clearing upper airways of the virus. Recent data have also suggested the treatment of COVID-19 patients with hydroxychloroquine and azithromycin to cure the associated infection. The objective of this trial is to assess the efficacy of standard of care (SOC) plus hydroxychloroquine monotherapy or SOC plus a combination of
\end{abstract}

\footnotetext{
* Correspondence: deborah.wallace@ucd.ie

${ }^{2}$ School of Medicine, University College Dublin, Dublin, Ireland

Full list of author information is available at the end of the article
}

hydroxychloroquine and azithromycin in subjects with non-critical, SARS-CoV-2 PCR-positive infection not requiring immediate resuscitation or ventilation but who have evidence of clinical decline compared to SOC alone.

\section{Trial design}

This is a multi-centre, prospective, randomised, open label, 3 arm (ratio 1:1:1) trial with parallel group design.

\section{Participants}

Patients with confirmed diagnosis of COVID-19 (PCR positive) who are non-critical but show evidence of progressive clinical decline at St. Vincent's University

(c) The Author(s). 2020 Open Access This article is licensed under a Creative Commons Attribution 4.0 International License, which permits use, sharing, adaptation, distribution and reproduction in any medium or format, as long as you give appropriate credit to the original author(s) and the source, provide a link to the Creative Commons licence, and indicate if changes were made. The images or other third party material in this article are included in the article's Creative Commons licence, unless indicated otherwise in a credit line to the material. If material is not included in the article's Creative Commons licence and your intended use is not permitted by statutory regulation or exceeds the permitted use, you will need to obtain permission directly from the copyright holder. To view a copy of this licence, visit http://creativecommons.org/licenses/by/4.0/ The Creative Commons Public Domain Dedication waiver (http://creativecommons.org/publicdomain/zero/1.0/) applies to the data made available in this article, unless otherwise stated in a credit line to the data. 
Hospital and Mater Misericordiae University Hospital, Dublin, Ireland. Inclusion Criteria Documented COVID19 positive (positive PCR test on a respiratory sample for SARS-CoV2), Aged $\geq 18$ years male or female. Evidence of progressive clinical decline as defined by: elevated and /or rising (over a 24hour period) inflammatory markers (at least two of in CRP, d-dimer, $\mathrm{LDH}$ and/or ferritin above the upper limit of normal), presence of or progression of pulmonary infiltrates on CXR (as decided by the treating physician), new hypoxia requiring $>21 / \mathrm{min} / 28 \% \mathrm{FiO} 2$ to maintain oxygen saturations $\geq 94 \%$ (or $88-92 \%$ in patients with chronic hypercapnic respiratory failure).

\section{Intervention and comparator}

Interventions for participants in this trial are SOC plus hydroxychloroquine monotherapy or SOC plus a combination of hydroxychloroquine and azithromycin. Following randomization, subjects will receive either (Arm 2) SOC plus hydroxychloroquine $(400 \mathrm{mg}$ BID on day 1 then $200 \mathrm{mg}$ twice daily from day 2 to 10 ) or (Arm 3) SOC plus hydroxychloroquine $(400 \mathrm{mg}$ BID on day 1 then $200 \mathrm{mg}$ twice daily from days 2 to 10 ) and azithromycin (500mg day 1 and 250mg daily from days 2 to 5 ). The comparator in this trial is SOC alone (Arm 1).

\section{Main outcomes}

The primary endpoint for this trial is a composite endpoint for time to progression to intubation, non-invasive ventilation, use of immunomodulatory therapy* for COVID-19 infection or death.

"Immunomodulatory therapy refers to use of high dose corticosteroids (methylprednisolone) or new initiation of any humanised monoclonal antibody or convalescent serum

\section{Randomisation}

Eligible patients (351) will be randomised using a central register in the ratio $1: 1: 1$, (117 per arm) with the use of permuted blocks of random sizes. To ensure concealment, the block sizes will not be disclosed. Randomisation will be performed through an interactive web-based electronic data capturing database.

\section{Blinding}

This study is open label. The study will not be blinded to investigators, subjects, or medical or nursing staff. The trial statistician will be blinded for data analysis and will be kept unaware of treatment group assignments. To facilitate this, the randomisation schedule will be drawn up by an independent statistician. Furthermore, we defined objective criteria for the primary outcome to minimize potential bias.

\section{Numbers to be randomised}

A total of 351 patients will be randomised; 117 participants into SOC plus hydroxychloroquine monotherapy, 117 into SOC plus a combination of hydroxychloroquine and azithromycin intervention group and 117 into SOC.

\section{Trial Status}

The COVIRL001 trial (Protocol version 1.4, 05 May 2020) will commence in May 2020 at St. Vincent's University Hospital and Mater Misericordiae University Hospital, Dublin, Ireland. Recruitment is proceeding with the aim to achieve the target sample size on or before October 2020.

\section{Trial registration}

The COVIRL001 trial is registered on 06 May 2020 under EudraCT number: 2020-001265-36 (https://www. clinicaltrialsregister.eu/ctr-search/search?query=covid-1 9+AND+University+College+Dublin) and Protocol identification: UCDCRC/20/01

\section{Full protocol}

The full protocol for COVIRL001 is attached as an additional file, accessible from the Trials website (Additional file 1). In the interest in expediting dissemination of this material, the familiar formatting has been eliminated; this Letter serves as a summary of the key elements of the full protocol (Protocol version 1.4, 05 May 2020). The study protocol has been reported in accordance with the Standard Protocol Items: Recommendations for Clinical Interventional Trials (SPIRIT) guidelines (Additional file 2).

\section{Keywords}

COVID-19; Randomised controlled trial; protocol; hydroxychloroquine; azithromycin

\section{Supplementary information}

Supplementary information accompanies this paper at https://doi.org/10 1186/s13063-020-04407-x.

Additional file 1. COVIRL-001_Protocol_V1.4.

Additional file 2. SPIRIT_COVIRL-001.

Additional file 3. COVIRL_PIL.

\section{Acknowledgements}

COVRIL001 Investigators: St Vincent's University Hospital, Silke Ryan, Alistair Nicholl, Marcus Butler, Charles Gallagher, Sarmad Waqas, Cathal O’Brion,

Stefano Savinelli, Mater Misericordiae University Hospital, Tara McGinty, Eavan Muldoon, Jack Lambert, Gerard Sheehan, Jim Egan, Sean Gaine, Brian

McCullough, Dermot O'Callaghan, UCD Centre for Experimental Pathogen Host Research, Alejandro Garcia Leon

Authors' contributions

$\mathrm{PM}, \mathrm{PD}, \mathrm{EF}, \mathrm{AC}, \mathrm{CMC}$ and $\mathrm{DK}$ contributed to the study concept and trial design, PM, PD, EF, AC, CMC, DK, DW, WT, EAB, RH contributed to the study protocol. The authors read and approved the final manuscript. 


\section{Funding}

University College Dublin is funding this trial and will have full oversight of the design of the study and collection, analysis, and interpretation of data and in writing the manuscript.

\section{Availability of data and materials}

Individual requests for access to the trial database will be considered in discussion with the local Research Ethics Committee.

\section{Ethics approval and consent to participate}

This trial has received ethical approval from the Research Ethics Committee at St. Vincent's University Hospital, Dublin, Ireland on 23 April 2020 (reference COVIRL-001). Written prospective informed consent will be obtained from participants prior to involvement in the trial (Additional file 3). University College Dublin, Clinical Research Centre, Dublin, Ireland is the sponsor for this study.

\section{Consent for publication}

Written, prospective informed consent will be obtained from all patients / subjects legally acceptable representative prior to inclusion in the trial for collection, storage, analysis and dissemination of the results of the trial.

\section{Competing interests}

The authors declare that they have no competing interests.

\section{Author details}

${ }^{1}$ St Vincent's University Hospital, Dublin, Ireland. ${ }^{2}$ School of Medicine, University College Dublin, Dublin, Ireland. ${ }^{3}$ Mater Misericordiae University Hospital, Dublin, Ireland. ${ }^{4}$ HRB-Trial Methodology Research Network, Galway, Ireland.

Received: 8 May 2020 Accepted: 11 May 2020

Published online: 25 May 2020

\section{Publisher's Note}

Springer Nature remains neutral with regard to jurisdictional claims in published maps and institutional affiliations. 